\title{
Variational projectors in fibred manifolds
}

Demeter Krupka, Zbyněk Urban, and Jana Volná 


\title{
VARIATIONAL PROJECTORS IN FIBRED MANIFOLDS
}

\author{
DEMETER KRUPKA, ZBYNĚK URBAN, AND JANA VOLNÁ
}

\begin{abstract}
The aim of this paper is to give a survey of recent developments in global variational geometry, and in particular, to complete the results on the construction of classes (terms) in the variational sequences related to higher-order variational problems on fibred spaces. Explicit description of the first order variational sequences is given as an example.
\end{abstract}

2010 Mathematics Subject Classification: 49Q99, 58A20, 58E30, 70 G75

Keywords: Jet bundle, Lagrangian, Euler-Lagrange expressions, Helmholtz conditions, variational sequence, variational projector.

\section{INTRODUCTION}

This paper contains the essential parts of the lecture presented by the first author at the workshop Algebra, Geometry and Mathematical Physics, Brno University of Technology, September 12-14, 2012, and prepared in collaboration with Zbyněk Urban and Jana Volná. The aim is to give a survey of the basic concepts and theorems of global variational geometry, and in particular to complete the construction of the terms (classes) in the variational sequences related to higher-order variational problems on fibred spaces (Section 3).

Our main references for general higher-order variational theory on smooth manifolds (Section 2) are the papers Garcia [4], Goldschmidt and Sternberg [5], Krupka [7], and Trautman [15], where the geometric setting for the theory of variational functionals has been established. Later, essential contributions were obtained by Anderson and Duchamp [2], and Dedecker [3]; a broad, conceptually more complete treatment, including invariance of variational functionals, was presented by Olver [14]. For contemporary status of the theory, we refer to the survey chapters in the book Krupka and Saunders [11], where many other relevant references can be found.

For the notion of the interior Euler-Lagrange operator, as defined by a chart expression, we refer to Anderson [1], and Krbek and Musilová [6] (see also other sources quoted therein). In global variational theory, we relate this notion to different terms (classes) in the variational sequence; to this purpose, however, the existing theory should be completed by proving the coordinate independence of the interior Euler-Lagrange operator, and then also by a description of its image. In this paper, we apply a coordinate-free definition given by Krupka and Šeděnková-Volná [13], and 
Volná and Urban [16], and extend the description of the interior Euler-Lagrange operators by the discussion of the image (Section 3 ). We also briefly discuss the classes entering the first order variational sequence in field theory. In order to avoid misunderstanding with the Euler-Lagrange mapping, we change terminology and call the (global version of) interior Euler-Lagrange operators the variational projectors.

From now on, we suppose we are given a fibred manifold $Y$ with base $X$ and projection $\pi$ and set $n=\operatorname{dim} X$ and $n+m=\operatorname{dim} Y$. We denote by $J^{r} Y$ the $r$ jet prolongation of $Y$. An element of the set $J^{r} Y$ is usually denoted by $J_{x}^{r} \gamma$; the mapping $x \rightarrow J^{r} \gamma(x)=J_{x}^{r} \gamma$ is the $r$-jet prolongation of $\gamma$. The canonical source projection $J_{x}^{r} \gamma \rightarrow x$ of $J^{r} Y$ onto $X$ (resp. the canonical jet projection $J_{x}^{r} \gamma \rightarrow J_{x}^{s} \gamma$ of $J^{r} Y$ onto $\left.J^{s} Y, 0 \leq s \leq r\right)$ is denoted by $\pi^{r}$ (resp. $\pi^{r, s}$ ). The $r$-jet prolongation $J^{r} \Xi$ of a $\pi$-projectable vector field $\Xi$, is a vector field on $J^{r} Y$ whose flow is the canonical jet prolongation of the flow of $\Xi$. For any open set $W \subset Y$, we set $W^{r}=$ $\left(\pi^{r, 0}\right)^{-1}(W)$ and denote by $\Omega^{r} W$ the exterior algebra of differential forms on the set $W^{r}$.

If $(V, \psi), \psi=\left(x^{i}, y^{\sigma}\right)$, is a fibred chart on $Y$, then the associated fibred chart on $J^{r} Y$ is denoted by $\left(V^{r}, \psi^{r}\right), \psi^{r}=\left(x^{i}, y^{\sigma}, y_{i_{1}}^{\sigma}, y_{i_{1} i_{2}}^{\sigma}, \ldots, y_{i_{1} i_{2} \ldots i_{r}}^{\sigma}\right)$. When we use a multi-index notation, we write $\psi^{r}=\left(x^{i}, y_{I}^{\sigma}\right)$, where $I=\left(i_{1} i_{2} \ldots i_{s}\right)$, and the length $s=|I|$ of $I$ satisfies $0 \leq|I| \leq r$. We also write $I i=\left(i_{1} i_{2} \ldots i_{s} i\right)$. Differential forms, locally generated by differential forms $\omega_{I}^{\sigma}=d y_{I}^{\sigma}-y_{I i}^{\sigma} d x^{i}, 0 \leq|I| \leq r-1$, and $d \omega_{I}^{\sigma},|I|=r-1$, are called contact forms. The canonical decomposition theorem (cf. e.g. Krupka [9]) for a $q$-form $\rho$ on $J^{r} Y$ says that $\left(\pi^{r+1, r}\right)^{*} \rho=h \rho+p_{1} \rho+$ $p_{2} \rho+\ldots+p_{q} \rho$, where the component $p_{k} \rho$ contains exactly $k$ exterior factors $\omega_{I}^{\sigma}$.

Throughout, standard concepts of analysis of differential forms on smooth manifolds are used; $d \rho$ is the exterior derivative of a form $\rho$, and $i_{\xi} \rho$ (resp. $\partial_{\xi} \rho$ ) is the contraction (resp. Lie derivative) of $\rho$ by a vector field $\xi$.

\section{VARIATIONAL FUNCTIONALS}

We introduce a morphism of exterior algebras related with a fibred manifold structure.

Lemma 1. Let $W$ be an open set in the fibred manifold $Y$. Then there exists a unique morphism $\Omega^{r} W \ni \rho \rightarrow h \rho \in \Omega^{r+1} W$ of exterior algebras, such that for any fibred chart $(V, \psi), \psi=\left(x^{i}, y^{\sigma}\right)$, with $V \subset W$, and any differentiable function $f: W^{r} \rightarrow \mathbb{R}$,

$$
h f=f \circ \pi^{r+1, r}, \quad h d f=d_{i} f \cdot d x^{i},
$$

where

$$
d_{i} f=\frac{\partial f}{\partial x^{i}}+\sum_{j_{1} \leq j_{2} \leq \ldots \leq j_{k}} \frac{\partial f}{\partial y_{j_{1} j_{2} \ldots j_{k}}^{\sigma}} y_{j_{1} j_{2} \ldots j_{k} i}^{\sigma} .
$$


The morphism $h$ is called the horizontalisation. The symbol $d_{i}$ in (2.2) is called the $i$-th formal derivative operator relative to the fibred chart $(V, \psi)$. It is important for practical calculations that $h d x^{i}=d x^{i}$ and $h d y_{I}^{\sigma}=y_{I i}^{\sigma} d x^{i}$.

A Lagrangian for $Y$ is a $\pi^{r}$-horizontal $n$-form $\lambda$ on the $r$-jet prolongation $J^{r} Y$ of $Y$. The number $r$ is the order of $\lambda$. The pair $(Y, \lambda)$ is sometimes called the variational structure. In a fibred chart $(V, \psi), \psi=\left(x^{i}, y^{\sigma}\right), \lambda$ has an expression $\lambda=\mathscr{L} \omega_{0}$, where $\omega_{0}=d x^{1} \wedge d x^{2} \wedge \ldots \wedge d x^{n}$ and $\mathscr{L}: V^{r} \rightarrow \mathbb{R}$ is the component of $\lambda$ with respect to $(V, \psi)$ (the Lagrange function).

Let $\Omega$ be a piece of $X$ with boundary $\partial \Omega$, let $\Gamma_{\Omega} Y$ be the set of sections of $Y$, defined on $\Omega$. A Lagrangian $\lambda$ of order $r$ defines the variational functional

$$
\Gamma_{\Omega} Y \ni \gamma \rightarrow \lambda_{\Omega}(\gamma)=\int_{\Omega} J^{r} \gamma^{*} \lambda \in \mathbb{R} .
$$

The main objective of the variational theory is to study the properties of this realvalued function, such as extremals and invariance conditions. To investigate $\lambda_{\Omega}$, we apply variational methods based on variations of each individual section $\gamma$ of $Y$, and induced variations of the value $\lambda_{\Omega}(\gamma)$. Given a $\pi$-projectable vector field $\Xi$ on $Y$, and its $\pi$-projection $\xi$, we can construct 1-parameter family of sections $\gamma_{t}=\alpha_{t}^{\Xi} \gamma \alpha_{-t}^{\xi}$, using the flows $\alpha_{t}^{\Xi}$ and $\alpha_{t}^{\xi}$ of $\Xi$ and $\xi$. Then, differentiating the function $t \rightarrow \lambda_{\Omega}\left(\gamma_{t}\right)$ at $t=0$, we get another variational functional

$$
\Gamma_{\Omega} Y \ni \gamma \rightarrow\left(\frac{d}{d t} \lambda_{\Omega}\left(\gamma_{t}\right)\right)_{0}=\int_{\Omega} J^{r} \gamma^{*} \partial_{J^{r} \Xi} \lambda \in \mathbb{R},
$$

the first variation of (2.3), induced by $\Xi$. For further computations of the Lie derivative $\partial_{J^{r} \Xi} \lambda$ in this expression, we need the following result.

Theorem 1 (Lepage equivalents of a Lagrangian). Let $\lambda$ be a Lagrangian of order $r$ for $Y$. There exists an integer $s$ and an $n$-form $\rho$ on $J^{s} Y$ such that:

(a) $J^{s} \gamma^{*} \rho=J^{r} \gamma^{*} \lambda$ for all sections $\gamma$ of $Y$.

(b) $J^{s} \gamma^{*} i_{\Xi} d \rho=0$ for all sections $\gamma$ of $Y$ and all $\pi^{s, 0}$-vertical vector fields $\Xi$ on $J^{s} Y$.

Conditions (a) and (b) can be equivalently expressed in terms of the horizontalisation $h$ as $h \rho=\lambda$ and $h i_{\Xi} d \rho=0$, respectively.

Any form $\rho$, satisfying conditions (a) and (b), is called a Lepage equivalent of the Lagrangian $\lambda$. Comparing condition (a) with formula (2.3), we see that a Lepage equivalent $\rho$ defines the same variational functional as $\lambda$.

There are many interesting examples of Lepage equivalents (see references in Krupka, Krupková and Saunders [12]). We mention here the principal Lepage equivalent of a second-order Lagrangian $\lambda=\mathscr{L} \omega_{0}$,

$$
\Theta=\mathscr{L} \omega_{0}+\left(\left(\frac{\partial \mathscr{L}}{\partial y_{i}^{\sigma}}-d_{j} \frac{\partial \mathscr{L}}{\partial y_{i j}^{\sigma}}\right) \omega^{\sigma}+\frac{\partial \mathscr{L}}{\partial y_{i j}^{\sigma}} \omega_{j}^{\sigma}\right) \wedge \omega_{i},
$$


where $\omega^{\sigma}=d y^{\sigma}-y_{l}^{\sigma} d x^{l}, \omega_{i}^{\sigma}=d y_{i}^{\sigma}-y_{i l}^{\sigma} d x^{l}$, and $\omega_{i}=i_{\partial / \partial x^{i}} \omega_{0}$ (Krupka [7]). The Lepage form $\Theta$ is important in applications. If $\lambda$ is of the first order, then $\Theta$ reduces to the well-known Poincaré-Cartan form introduced by Garcia [4].

Theorem 2 (Infinitesimal first variation formula). Let $\lambda$ be a Lagrangian of order $r$ for $Y$, and let $\rho$ be any Lepage equivalent of $\lambda$, defined on $J^{s} Y$. Then, for any section $\gamma$ and any $\pi$-projectable vector field $\Xi$ on $Y$,

$$
J^{r} \gamma^{*} \partial_{J^{r} \Xi} \lambda=J^{s} \gamma^{*} i_{J^{s} \Xi} d \rho+d J^{s} \gamma^{*} i_{J^{s} \Xi} \rho .
$$

Formula (2.6) is called the infinitesimal first variation formula. It corresponds with the integrand of the "classical" (integral) first variation formula, but does not have a classical analogue. Using the horizontalisation, (2.6) can be equivalently written as

$$
\partial_{J^{r} \Xi} \lambda=h i_{J^{s} \Xi} d \rho+h d i_{J^{s} \Xi} \rho .
$$

The Euler-Lagrange form of a Lagrangian $\lambda$ of order $r$ is an $(n+1)$-form $E_{\lambda}$ on $J^{2 r} Y$, defined by

$$
E_{\lambda}=E_{\sigma}(\mathscr{L}) \omega^{\sigma} \wedge \omega_{0},
$$

where $E_{\sigma}(\mathscr{L})$ are the Euler-Lagrange expressions,

$$
E_{\sigma}(\mathscr{L})=\sum_{l=0}^{r}(-1)^{l} d_{p_{1}} d_{p_{2}} \ldots d_{p_{l}} \frac{\partial \mathscr{L}}{\partial y_{p_{1} p_{2} \ldots p_{l}}^{\sigma}} .
$$

It can be verified that the first summand on the right-hand side of (2.6) represents the Euler-Lagrange form: by a direct computation $J^{s} \gamma^{*} i_{J^{s} \Xi} d \rho=J^{s+1} \gamma^{*} i_{J^{s+1} \Xi} E_{\lambda}$. The boundary term $d J^{s} \gamma^{*} i_{J^{s}} \rho$ is an exact differential form; in the integral first variation formula, it transforms, via the Stokes' theorem, to the boundary $\partial \Omega$.

Suppose that the Euler-Lagrange form $E_{\lambda}$ is of order $s$. Then a section $\gamma$ is called an extremal, if $E_{\lambda}$ vanishes along the $s$-jet prolongation $J^{s} \gamma ; \gamma$ is an extremal if and only if its restriction to any coordinate neighborhood satisfies the Euler-Lagrange equations $E_{\sigma}(\mathscr{L})=0$.

The $\mathbb{R}$-linear mapping $\lambda \rightarrow E_{\lambda}$, assigning to a Lagrangian its Euler-Lagrange form, is called the Euler-Lagrange mapping. The Lagrangians, belonging to its kernel, are called (variationally) trivial, or null.

Theorem 3 (Trivial Lagrangians). For a Lagrangian $\lambda$ of order $r$, the following two conditions are equivalent:

(a) $E_{\lambda}=0$

(b) To each point $y_{0} \in Y$, there exist a fibred chart $(V, \psi)$ at $y_{0}$ and an $(n-1)$-form $\eta$ defined on $V^{r-1} \subset J^{r-1} Y$ such that

$$
J^{r} \gamma^{*} \lambda=d J^{r-1} \gamma^{*} \eta
$$

for all sections $\gamma$, defined on $U=\pi(V) \subset X$. 
Condition (b) is equivalent to the equation $\lambda=h d \eta$ on $V^{r}$.

By a source form on $J^{s} Y$, we mean a differential $(n+1)$-form $\varepsilon$ expressible in any fibred chart $(V, \psi), \psi=\left(x^{i}, y^{\sigma}\right)$, as

$$
\varepsilon=\varepsilon_{\sigma} \omega^{\sigma} \wedge \omega_{0} .
$$

Clearly, this definition is invariant under transformations of fibred coordinates. Every Euler-Lagrange form is a source form. A source form is said to be variational if it is expressible as $\varepsilon=E_{\lambda}$ for some Lagrangian $\lambda$. The problem as to find conditions under which a source form is variational is the inverse problem of the calculus of variations.

To study the invariant variational structure, let us first mention, for the record, some general differential-geometric notions that are needed, sometimes with slight modifications, in proofs. Let $X$ be a manifold, $W$ an open set in $X$ and $\alpha: W \rightarrow X$ a diffeomorphism, let $\rho$ be a $p$-form on $X$. We say that $\rho$ is invariant with respect to $\alpha$, if its pull-back $\alpha^{*} \rho$ coincides with $\rho$,

$$
\alpha^{*} \rho=\rho .
$$

We also say that $\alpha$ is an invariance transformation of $\rho$. This definition immediately extends to vector fields. Let $\xi$ be a vector field on $X, \alpha^{\xi}$ its flow and $\alpha_{t}^{\xi}$ its local 1-parameter group. $\xi$ is said to be the generator of invariance transformations of $\rho$ if for all $(t, x)$ from the domain of the flow $\alpha^{\xi}$,

$$
\left(\alpha_{t}^{\xi}\right)^{*} \rho(x)=\rho(x) .
$$

Lemma 2. For every point $(t, x)$ from the domain of the definition of the flow of the vector field $\xi$,

$$
\frac{d}{d t}\left(\alpha_{t}^{\xi}\right)^{*} \rho(x)=\left(\left(\alpha_{t}^{\xi}\right)^{*} \partial_{\xi} \rho\right)(x)
$$

Lemma 3. The following two conditions are equivalent:

(a) $\xi$ generates invariance transformations of $\rho$.

(b) The Lie derivative of $\rho$ by $\xi$ vanishes,

$$
\partial_{\xi} \rho=0 .
$$

Let $\lambda$ be a Lagrangian of order $r$ for $Y, \alpha: W \rightarrow Y$ an automorphism of the fibred manifold $Y$, and $J^{r} \alpha: W^{r} \rightarrow J^{r} Y$ the $r$-jet prolongation of $\alpha$. We say that $\alpha$ is an invariance transformation of $\lambda$, if $\left(J^{r} \alpha\right)^{*} \lambda=\lambda$. A generator of invariance transformations of $\lambda$ is a $\pi$-projectable vector field $\Xi$ on $Y$ whose local one-parameter group consists of invariance transformations of $\lambda$. The definitions naturally extend to $r$-jet prolongations $J^{r} \Xi$ of $\Xi$.

In the following lemma, we use the multi-index notation. We introduce a class of vector fields on $J^{r} Y$, preserving the contact forms $\omega_{I}^{\sigma}=d y_{I}^{\sigma}-y_{I i}^{\sigma} d x^{i}, 0 \leq|I| \leq$ $r-1$. We say that a vector field $Z$ on $J^{r} Y$ preserves contact forms if, for any contact 
form $\rho$, the Lie derivative $\partial_{Z} \rho$ is again a contact form; we also say that $Z$ is a contact symmetry (see Krupka, Krupkova, Prince and Sarlet [10]).

Lemma 4. For any $\pi$-projectable vector field $\Xi$ on $Y$, the $r$-jet prolongation $Z=J^{r} \Xi$ is a contact symmetry.

Lemma 5. Let $\lambda$ be a Lagrangian of order $r$ for $Y$.

(a) A $\pi$-projectable vector field $\Xi$ on $Y$ generates invariance transformations of $\lambda$ if and only if

$$
\partial_{J^{r}} \Xi \lambda=0
$$

(b) Generators of invariance transformations of $\lambda$ constitute a subalgebra of the algebra of the vector fields on $J^{r} Y$.

In terminology used by Trautman [15] (see also Krupka [8]), equation (2.16) is the Noether equation. This equation represents a relation between the Lagrangian $\lambda$ and the generator $\Xi$ of invariance transformations. Given $\lambda$, we can use the Noether equation to determine the generators $\Xi$. On the other hand, given a Lie algebra of $\pi$-projectable vector fields $\Xi$, one can use the corresponding Noether equations to determine invariant Lagrangians $\lambda$.

Theorem 4. Suppose that a Lagrangian $\lambda$ is invariant with respect to a $\pi$-projectable vector field $\Xi$. Then for any Lepage equivalent $\rho$ of order $s$ of $\lambda$,

$$
J^{s} \gamma^{*} i_{J^{s} \Xi} d \rho+d J^{s} \gamma^{*} i_{J^{s} \Xi} \rho=0
$$

for every section $\gamma$ of $Y$.

By a conserved current for a section $\gamma \in \Gamma_{\Omega}(Y)$, we mean any $(n-1)$-form $\eta$ on $J^{r} Y$ such that

$$
d J^{r} \gamma^{*} \eta=0 .
$$

We call formula (2.18) the conservation law equation, or a conservation law for the section $\gamma$.

The following assertion says that extremals of invariant Lagrangians satisfy, in addition to the Euler-Lagrange equations, also some other conditions in the form of conservation law equations.

Theorem 5 (First theorem of Emmy Noether). Let $\lambda$ be a Lagrangian, $\rho$ a Lepage equivalent of $\lambda$ defined on $J^{s} Y$ and let $\gamma$ be an extremal. Then, for every generator $\Xi$ of invariance transformations of $\lambda$,

$$
d J^{s} \gamma^{*} i_{J_{\Xi}} \rho=0 .
$$

One can use invariance of variational functionals in a different way than in Theorem 5. Namely, the infinitesimal first variation formula (Theorem 2) shows that the property of a Lagrangian to be invariant reduces the number of Euler-Lagrange equations. 
Theorem 6. Let $\lambda$ be an invariant Lagrangian with respect to a vector field $\Xi$, and suppose that a section $\gamma$ satisfies the conservation law

$$
d J^{s} \gamma^{*} i_{J^{s} \Xi} \rho=0
$$

for a Lepage equivalent $\rho$ of $\lambda$ of order $s$. Then, for any fibred chart $(V, \psi)$, the associated Euler-Lagrange expressions are linearly dependent along $\gamma$.

Let $\alpha: W \rightarrow Y$ be an automorphism of $Y$ and let $\varepsilon$ be a source form on $J^{s} Y$. We say that $\alpha$ is an invariance transformation of $\varepsilon$, if $J^{s} \alpha^{*} \varepsilon=\varepsilon$. A generator of invariance transformations of $\varepsilon$ is a $\pi$-projectable vector field on $Y$ whose local one-parameter group consists of invariance transformations of $\varepsilon$.

Lemma 6 (Noether-Bessel-Hagen equation). Let $\varepsilon$ be a source form of order $s$ for $Y$.

(a) A $\pi$-projectable vector field $\Xi$ on $Y$ is the generator of invariance transformations of $\varepsilon$ if and only if

$$
\partial_{J^{r} \Xi} \varepsilon=0
$$

(b) Generators of invariance transformations of $\varepsilon$ constitute a subalgebra of the algebra of vector fields on $J^{r} Y$.

To study invariant Euler-Lagrange forms, note the following identity.

Lemma 7. Let $\lambda$ be a Lagrangian of order $r$ for $Y$, let $\alpha$ be an automorphism of $Y$, and let $E_{\lambda}$ be the Euler-Lagrange form of $\lambda$. Then

$$
J^{2 r} \alpha^{*} E_{\lambda}=E_{J^{r} \alpha^{*} \lambda} .
$$

Lemma 8. (a) Every invariance transformation of a Lagrangian $\lambda$ is an invariance transformation of the Euler-Lagrange form $E_{\lambda}$.

(b) For every invariance transformation $\alpha$ of $E_{\lambda}$, the Lagrangian $\lambda-J^{r} \alpha^{*} \lambda$ is variationally trivial.

We can generalize Noether's theorem to invariance transformations of the EulerLagrange forms and conservation equations. However, since the proof is based on the theorem on the kernel of the Euler-Lagrange mapping $\lambda \rightarrow E_{\lambda}$, which is of local character (Theorem 3), the assertion we obtain is also of local character. Let $\Theta_{\lambda}$ denote the principal Lepage equivalent of $\lambda$ (see Krupka, Krupková, Saunders [12]); for second-order Lagrangians, $\Theta_{\lambda}$ was defined in (2.5). Note, however, that for general orders $\Theta_{\lambda}$ is not necessarily defined globally.

Theorem 7. Let $\lambda$ be a Lagrangian of order $r, \gamma$ an extremal, and $\Xi$ the generator of invariance transformations of the Euler-Lagrange form $E_{\lambda}$. Then, for every point $y_{0} \in Y$, there exists a fibred chart $(V, \psi)$ at $y_{0}$ and an $(n-1)$-form $\eta$ defined on $V^{r-1}$ such that

$$
d J^{2 r} \gamma^{*}\left(i_{J^{2 r} \Xi} \Theta_{\lambda}-\eta\right)=0
$$

on $U=\pi(V)$. 
Now, we study symmetries of solutions of the Euler-Lagrange equations. Let $\lambda$ be a Lagrangian of order $r$ for $Y$. Suppose that we have an extremal $\gamma$ of $\lambda$; thus, $\gamma$ satisfies the Euler-Lagrange equations

$$
E_{\lambda} \circ J^{2 r} \gamma=0 .
$$

An automorphism $\alpha: W \rightarrow Y$ of $Y$ with projection $\alpha_{0}$ is said to be a symmetry of $\gamma$, if the section $\alpha \gamma \alpha_{0}^{-1}$ is also an extremal, i.e.,

$$
E_{\lambda} \circ J^{2 r}\left(\alpha \gamma \alpha_{0}^{-1}\right)=0 \text {. }
$$

We say that a $\pi$-projectable vector field $\Xi$ generates symmetries of $\gamma$ if its local one-parameter group consists of symmetries of $\gamma$.

To study symmetries, we need a lemma on pushforward vector fields. Let $X$ be any manifold, $\xi$ a vector field on $X, W \subset X$ an open set, and $\alpha: W \rightarrow X$ a diffeomorphism. The pushforward vector field of $\xi$ by $\alpha$ is the vector field

$$
\xi^{(\alpha)}(x)=T_{\alpha^{-1}(x)} \alpha \cdot \xi\left(\alpha^{-1}(x)\right) .
$$

Lemma 9. Let $X$ be a manifold, $W$ an open set in $X$, $\xi$ a vector field on $X$, $\alpha: W \rightarrow X$ a diffeomorphism, and $\rho$ a $p$-form. Then

$$
i_{\xi} \alpha^{*} \rho=\alpha^{*} i_{\xi(\alpha)} \rho .
$$

The following theorem says that invariance transformations of the Euler-Lagrange form $E_{\lambda}$ permute extremals of the variational structure $(Y, \lambda)$ and give us examples of symmetries of extremals.

Theorem 8. An invariance transformation of the Euler-Lagrange form $E_{\lambda}$ is a symmetry of every extremal $\gamma$ of $\lambda$.

The following theorem describes the properties of individual extremals.

Theorem 9. Let $\lambda$ be a Lagrangian of order $r$, let $s$ be the order of the EulerLagrange form $E_{\lambda}$, and let $\gamma$ be an extremal. Then a $\pi$-projectable vector field $\Xi$ generates symmetries of $\gamma$ if and only if

$$
E_{\partial_{J}{ }_{\Xi} \lambda} \circ J^{s} \gamma=0 .
$$

\section{VARIATIONAL SEQUENCES AND VARIATIONAL PROJECTORS}

We have already defined contact forms on the $r$-jet prolongation $J^{r} Y$. Evidently, every $q$-form, where $q \geq n+1, n=\operatorname{dim} X$, would be contact. Now, we introduce, for $(n+k)$-forms, $k \geq 1$, a modified concept of a strongly contact form. We say that an $(n+k)$-form $\rho$ on $J^{r} Y$ is strongly contact if, for every point $J_{x}^{r} \gamma \in J^{r} Y$, there exist a fibred chart $(V, \psi), \psi=\left(x^{i}, y^{\sigma}\right)$, on $Y$, an integer $s \geq r$, and a contact $(n+k-1)$-form $\eta$ on $V^{s}$ with $p_{k-1} \eta=0$ such that

$$
p_{k}\left(\left(\pi^{s, r}\right)^{*} \rho-d \eta\right)=0 .
$$


This condition is equivalent to saying that $\left(\pi^{s, r}\right)^{*} \rho$ can be expressed in the form

$$
\left(\pi^{s, r}\right)^{*} \rho=\mu+d \eta,
$$

where $\mu$ and $\eta$ satisfy $p_{k} \mu=0$ and $p_{k-1} \eta=0$.

Let $q \geq 0, k \geq 1$. If $0 \leq q \leq n$, denote by $\Theta_{q}^{r}$ the sheaf of contact forms on $J^{r} Y$; if $q>n$, then let $\Theta_{q}^{r}$ be the sheaf of strongly contact forms on $J^{r} Y$. We get an exact subsequence

$$
0 \rightarrow \Theta_{1}^{r} \rightarrow \Theta_{2}^{r} \rightarrow \Theta_{3}^{r} \rightarrow \ldots \rightarrow \Theta_{M}^{r} \rightarrow 0
$$

of the de Rham sequence of sheaves of Abelian groups on $J^{r} Y$,

$$
0 \rightarrow \mathbb{R} \rightarrow \Omega_{0}^{r} \rightarrow \Omega_{1}^{r} \rightarrow \Omega_{2}^{r} \rightarrow \ldots \rightarrow \Omega_{M}^{r} \rightarrow \Omega_{M+1}^{r} \rightarrow \ldots \rightarrow \Omega_{N}^{r} \rightarrow 0,
$$

with the sequence mappings equal to the exterior derivative operator $d$. Then numbers $N$ and $M$ are given by

$$
N=\operatorname{dim} J^{r} Y, \quad M=n\left(\begin{array}{c}
n+r-1 \\
n
\end{array}\right)+2 n-1 .
$$

We call (3.3) the contact subsequence of the de Rham sequence. The quotient sequence

$$
0 \rightarrow \mathbb{R} \rightarrow \Omega_{0}^{r} \rightarrow \Omega_{1}^{r} / \Theta_{1}^{r} \rightarrow \ldots \rightarrow \Omega_{M}^{r} / \Theta_{M}^{r} \rightarrow \Omega_{M+1}^{r} \rightarrow \ldots \rightarrow \Omega_{N}^{r} \rightarrow 0
$$

is also exact; we call the quotient sequence the variational sequence (of order $r$ ). The class $[\rho]$ of a form $\rho \in \Omega_{n}^{r}\left(\rho \in \Omega_{n+1}^{r}, \rho \in \Omega_{n+2}^{r}\right)$ is called the Lagrangian (Euler-Lagrange class, Helmholtz class) of $\rho$. The quotient mappings $E: \Omega_{k}^{r} / \Theta_{k}^{r} \rightarrow$ $\Omega_{k+1}^{r} / \Theta_{k+1}^{r}$ are defined by

$$
E([\rho])=[d \rho] .
$$

The mapping $E: \Omega_{n}^{r} / \Theta_{n}^{r} \rightarrow \Omega_{n+1}^{r} / \Theta_{n+1}^{r}$, assigning to a Lagrangian $\lambda=[\rho]$ its Euler-Lagrange class $E(\lambda)=[d \rho]$, is called the Euler-Lagrange mapping; one can extend the definition and call each of the quotient mappings in (3.6) the EulerLagrange morphisms.

Theorem 10. The variational sequence is an acyclic resolution of the constant sheaf $\mathbb{R}$.

In the well-known sense, Theorem 10 allows us to identify the cohomology of the sequence of global sections of (3.6) with the De Rham cohomology of the underlying fibred manifold $Y$ (see Krupka [9]). In particular, we get cohomology conditions for the existence of global variational principles for systems of differential equations on a manifold, expressed by means of a source form.

Now, we explain without proofs the basic results on variational projectors (Volná and Urban [16]).

Since our definition of the variational projectors is inductive, we first need the following lemma on the structure of 1-contact $(n+1)$-forms. 
Lemma 10. Let $\rho$ be a 1-contact $\pi^{r+1, r}$-horizontal $(n+1)$-form on $W^{r+1} \subset$ $J^{r+1} Y$ expressed in a fibred chart $(V, \psi), \psi=\left(x^{i}, y^{\sigma}\right)$, by

$$
\rho=\sum_{0 \leq|J| \leq r} A_{\sigma}^{J} \omega_{J}^{\sigma} \wedge \omega_{0} .
$$

Then, there exist a unique 1 -contact $\omega^{\sigma}$-generated $(n+1)$-form $I_{1} \rho$ on $W^{2 r+1} \subset$ $J^{2 r+1} Y$, and a 1 -contact $n$-form $J_{1} \rho$ and a 2 -contact $(n+1)$-form $K_{1} \rho$ defined over the chart neighborhood $V$ such that

$$
\left(\pi^{2 r+1, r+1}\right)^{*} \rho=I_{1} \rho-d J_{1} \rho+K_{1} \rho,
$$

where

$$
\begin{aligned}
& I_{1} \rho=B_{\sigma} \omega^{\sigma} \wedge \omega_{0}, \\
& J_{1} \rho=\sum_{0 \leq|J| \leq r-1} B_{\sigma}^{J i} \omega_{J}^{\sigma} \wedge \omega_{i}, \quad K_{1} \rho=\sum_{0 \leq|J| \leq r-1} p d B_{\sigma}^{J i} \wedge \omega_{J}^{\sigma} \wedge \omega_{i},
\end{aligned}
$$

and

$$
\begin{aligned}
B_{\sigma} & =\sum_{0 \leq s \leq r}(-1)^{s} d_{i_{1}} d_{i_{2}} \ldots d_{i_{s}} A_{\sigma}^{i_{1} i_{2} \ldots i_{s}}, \\
B_{\sigma}^{J i} & =\sum_{1 \leq q \leq r-k}(-1)^{q+1} d_{i_{k+2}} d_{i_{k+3}} \ldots d_{i_{k+q}} A_{\sigma}^{J i i_{k+2} i_{k+3} \ldots i_{k+q}},
\end{aligned}
$$

where $|J|=k=0,1,2, \ldots, r-1$.

The form $I_{1} \rho$, defined by Lemma 10 on $(n+1)$-forms $\rho$ in a unique way, can be extended to arbitrary $(n+k)$-forms. We proceed as follows.

Let $k>1$. Let $W$ be an open subset of $Y$. Suppose we have a $k$-contact $(n+k)$ form $\rho$ defined on $W^{r+1} \subset J^{r+1} Y$. Let $\Xi_{1}, \Xi_{2}, \ldots, \Xi_{k}$ be arbitrary $\pi$-vertical vector fields on $W \subset Y$. Then, we define a $k$-contact $(n+k)$-form $I_{k} \rho$ on $J^{2 r+1} Y$ by

$$
\begin{aligned}
i_{J^{2 r+1} \Xi_{k}} & \ldots i_{J^{2 r+1} \Xi_{2}} i_{J^{2 r+1} \Xi_{1}} I_{k} \rho \\
& =\frac{1}{k}\left(i_{J^{2 r+1} \Xi_{k}} i_{J^{2 r+1} \Xi_{k-1}} \ldots i_{J^{2 r+1} \Xi_{3}} i_{J^{2 r+1} \Xi_{2}} I_{k-1}\left(i_{J^{r+1}} \Xi_{1} \rho\right)\right. \\
& -i_{J^{2 r+1} \Xi_{k}} i_{J^{2 r+1} \Xi_{k-1}} \ldots i_{J^{2 r+1} \Xi_{3}} i_{J^{2 r+1} \Xi_{1}} I_{k-1}\left(i_{J^{r+1}} \Xi_{2} \rho\right) \\
& -i_{J^{2 r+1} \Xi_{k}} i_{J^{2 r+1} \Xi_{k-1}} \ldots i_{J^{2 r+1} \Xi_{4}} i_{J^{2 r+1} \Xi_{1}} i_{J^{2 r+1} \Xi_{2}} I_{k-1}\left(i_{J^{r+1}} \Xi_{3} \rho\right) \\
& -\ldots \\
& -i_{J^{2 r+1} \Xi_{k}} i_{J^{2 r+1} \Xi_{1}} i_{J^{2 r+1} \Xi_{k-2}} \ldots i_{J^{2 r+1} \Xi_{3}} i_{J^{2 r+1} \Xi_{2}} I_{k-1}\left(i_{J^{r+1} \Xi_{k-1}} \rho\right) \\
& \left.-i_{J^{2 r+1} \Xi_{1}} i_{J^{2 r+1} \Xi_{k-1}} \ldots i_{J^{2 r+1} \Xi_{3}} i_{J^{2 r+1} \Xi_{2}} I_{k-1}\left(i_{J^{r+1}} \Xi_{k} \rho\right)\right)
\end{aligned}
$$

and an $\mathbb{R}$-linear mapping $\Omega_{n+k}^{r} W \ni \rho \rightarrow \mathscr{I} \rho \in \Omega_{n+k}^{2 r+1} W$ by

$$
\mathscr{I} \rho=I_{k} p_{k} \rho \text {. }
$$


It should be pointed out that the form $\mathscr{I} \rho \in \Omega_{n+k}^{2 r+1} W$ is defined over the same open set $W$ as $\rho$. The following theorem has a fundamental meaning for applications of the theory of variational sequences.

Theorem 11. Let $k \geq 1$ be an integer. Let $\pi: Y \rightarrow X$ be a fibred manifold over $n$-dimensional manifold $X$, and let $W$ be an open set in $Y$.

(a) For every form $\rho \in \Omega_{n+k}^{r} W, \mathscr{I} \rho$ belongs to the same class as $\left(\pi^{2 r+1, r}\right)^{*} \rho$.

(b) The kernel of the mapping $\Omega_{n+k}^{r} W \ni \rho \rightarrow \mathscr{I} \rho \in \Omega_{n+k}^{2 r+1} W$ coincides with the Abelian group $\Theta_{n+k}^{r} W$.

(c) $\mathscr{I}$ satisfies, up to the canonical jet projection,

$$
\mathscr{I} \circ \mathscr{I}=\mathscr{I} .
$$

Remark. Theorem 11,(b) states that the kernel $\operatorname{Ker} \mathscr{I}$ of the morphism of Abelian groups $\Omega_{n+k}^{r} W \ni \rho \rightarrow \mathscr{I} \rho \in \Omega_{n+k}^{2 r+1} W$ coincides with the subgroup $\Theta_{n+k}^{r} W \subset$ $\Omega_{n+k}^{r} W$ of contact forms. Consequently, the image $\operatorname{Im} \mathscr{I}$ is canonically isomorphic with the quotient group $\Omega_{n+k}^{r} W / \Theta_{n+k}^{r} W$. Under this isomorphism, the class [ $\rho$ ] of a form $\rho \in \Omega_{n+k}^{r} W$ can be canonically identified with a form $\mathscr{I} \rho \in \Omega_{n+k}^{2 r+1} W$. In particular, the quotient group can be characterized as a subgroup of the group $\Omega_{n+k}^{2 r+1} W$; this gives a way to compute the classes. One can find equations of the subgroups; however, in general, an explicit solution of these equations is still an open problem.

\section{EXAMPLE: FIRST ORDER VARIATIONAL SEQUENCE IN FIELD THEORY}

We give some remarks on the structure of classes in the variational sequence on $J^{1} Y$ over an $n$-dimensional base. These expressions allow us to study the local inverse problem for systems of differential equations and are also related with the meaning of cohomological conditions arising from the variational sequence theory.

(a) Classes of $n$-forms (Lagrangians)

The class of a form $\rho \in \Omega_{n}^{1} W$ is canonically identified with an $n$-form $h \rho \in \Omega_{n}^{2} W$. Conversely, an $n$-form $\lambda \in \Omega_{n}^{2} W$ is a class if and only if the equation $\lambda=h \rho$ has a solution $\rho$. One can show that $\lambda$ is a class if and only if $\lambda=\mathscr{L} \omega_{0}$, where $\mathscr{L}$ is a polynomial

$$
\begin{aligned}
& \mathscr{L}=\mathscr{L}_{0}+P_{\sigma_{1}}^{j_{1} i_{1}} y_{j_{1} i_{1}}^{\sigma_{1}}+P_{\sigma_{1}}^{j_{1} i_{1}} j_{\sigma_{2}} i_{2} y_{j_{1} i_{1}}^{\sigma_{1}} y_{j_{2} i_{2}}^{\sigma_{2}}+ \\
& \ldots+P_{\sigma_{1}}^{j_{1} i_{1} j_{2} i_{2}} \ldots{ }_{\sigma_{n}}^{j_{n} i_{n}} y_{j_{1} i_{1}}^{\sigma_{1}} y_{j_{2} i_{2}}^{\sigma_{2}} \ldots y_{j_{n} i_{n}}^{\sigma_{n}}
\end{aligned}
$$

with the coefficients depending on $x^{i}, y^{v}, y_{j}^{v}$, symmetric in $j_{l}, i_{l}$, and such that $P_{\sigma_{1}}^{j_{1} i_{1} j_{\sigma_{2}} i_{2} \ldots \sigma_{k}} \ldots j_{k} i_{k}=0$ whenever one cannot choose $i_{1}, i_{2}, \ldots, i_{k}$ mutually different. 
(b) Classes of $(n+k)$-forms (source forms)

The class of an $(n+1)$-form $\rho \in \Omega_{n+1}^{1} W$ is canonically identified with a form $\mathscr{I} \rho \in \Omega_{n+1}^{3} W$, and conversely, an $(n+1)$-form $\varepsilon \in \Omega_{n+1}^{3} W$ is a class if and only if the equation $\varepsilon=\mathscr{I} \rho$ has a solution $\rho$ or, which is the same, $\varepsilon$ should satisfy integrability conditions for this equation. Proceeding in the same way as in (a), one can describe these conditions explicitly. The resulting expression for $\varepsilon$ is polynomial in $y_{j i}^{\sigma}$ and $y_{j i k}^{\sigma}$ whose coefficients should satisfy a system of first order partial differential equations. The classes of $(n+k)$-forms for general $k$ have the same structure.

\section{(c) The Euler-Lagrange mapping}

The mapping $E$ of the quotient group $\Omega_{n}^{1} W / \Theta_{n}^{1} W$ into $\Omega_{n+1}^{1} W / \Theta_{n+1}^{1} W$ is expressed by familiar formulas with some restrictions. Writing a class $\lambda$ as above, we get the corresponding class as an $(n+1)$-form $E(\lambda)=E_{\sigma}(\mathscr{L}) \omega^{\sigma} \wedge \omega_{0}$, where

$$
E_{\sigma}(\mathscr{L})=\frac{\partial \mathscr{L}}{\partial y^{\sigma}}-d_{i} \frac{\partial \mathscr{L}}{\partial y_{i}^{\sigma}}+d_{i} d_{j} \frac{\partial \mathscr{L}}{\partial y_{i j}^{\sigma}} .
$$

In addition, however, one should substitute for $\mathscr{L}$ from (4.1).

(d) The Helmholtz form

Given a source form $\varepsilon \in \Omega_{n+1}^{3} W$ such that $\varepsilon=\mathscr{I} \rho$ (i.e., belonging to the quotient group $\left.\Omega_{n+1}^{1} W / \Theta_{n+1}^{1} W\right)$, then the class $E(\varepsilon)=\mathscr{I} d \rho$ belongs to $\Omega_{n+2}^{1} W / \Theta_{n+2}^{1} W$, and it can equivalently be characterized as a form from the space of forms $\Omega_{n+2}^{3} W$. Writing $\varepsilon=\varepsilon_{\sigma} \omega^{\sigma} \wedge \omega_{0}$, we get a globally defined $(n+2)$-form

$$
\begin{aligned}
E(\varepsilon)=\left(H_{\sigma \nu}(\varepsilon) \omega^{v}+H_{\sigma \nu}^{p_{1}}(\varepsilon) \omega_{p_{1}}^{v}\right. & +H_{\sigma \nu}^{p_{1} p_{2}}(\varepsilon) \omega_{p_{1} p_{2}}^{v} \\
& \left.+H_{\sigma \nu}^{p_{1} p_{2} p_{3}}(\varepsilon) \omega_{p_{1} p_{2} p_{3}}^{\nu}\right) \wedge \omega^{\sigma} \wedge \omega_{0},
\end{aligned}
$$

where the components are the Helmholtz expression

$$
\begin{aligned}
H_{\sigma v}(\varepsilon) & =\frac{\partial \varepsilon_{\sigma}}{\partial y^{v}}-\frac{\partial \varepsilon_{v}}{\partial y^{\sigma}}+d_{p_{1}} \frac{\partial \varepsilon_{v}}{\partial y_{p_{1}}^{\sigma}}-d_{p_{1}} d_{p_{2}} \frac{\partial \varepsilon_{v}}{\partial y_{p_{1} p_{2}}^{\sigma}}+d_{p_{1}} d_{p_{2}} d_{p_{3}} \frac{\partial \varepsilon_{v}}{\partial y_{p_{1} p_{2} p_{3}}^{\sigma}}, \\
H_{\sigma \nu}^{p_{1}}(\varepsilon) & =\frac{\partial \varepsilon_{\sigma}}{\partial y_{p_{1}}^{v}}+\frac{\partial \varepsilon_{v}}{\partial y_{p_{1}}^{\sigma}}-2 d_{p_{2}} \frac{\partial \varepsilon_{v}}{\partial y_{p_{1} p_{2}}^{\sigma}}+3 d_{p_{2}} d_{p_{3}} \frac{\partial \varepsilon_{v}}{\partial y_{p_{1} p_{2} p_{3}}^{\sigma}}, \\
H_{\sigma \nu}^{p_{1} p_{2}}(\varepsilon) & =\frac{\partial \varepsilon_{\sigma}}{\partial y_{p_{1} p_{2}}^{v}}-\frac{\partial \varepsilon_{v}}{\partial y_{p_{1} p_{2}}^{\sigma}}+3 d_{p_{3}} \frac{\partial \varepsilon_{v}}{\partial y_{p_{1} p_{2} p_{3}}^{\sigma}}, \\
H_{\sigma \nu} p_{1} p_{2} p_{3}(\varepsilon) & =\frac{\partial \varepsilon_{\sigma}}{\partial y_{p_{1} p_{2} p_{3}}^{v}}+\frac{\partial \varepsilon_{v}}{\partial y_{p_{1} p_{2} p_{3}}^{\sigma}},
\end{aligned}
$$

with $\varepsilon$ satisfying the integrability condition for the equation $\varepsilon=\mathscr{I} \rho$. 


\section{ACKNOWLEDGEMENT}

The first author (DK) acknowledges the support of the National Science Foundation of China (project No. 109320020), and is grateful to the members and students of the School of Mathematics, Beijing Institute of Technology, for kind hospitality and collaboration during his work in China. He also appreciates the support from Grant 201/09/0981 of the Czech Science Foundation and from the IRSES project GEOMECH (Project No. 246981) within the 7th European Community Framework Programme. The second author (ZU) appreciates the support at the University of Pardubice from the Project CZ.1.07/2.3.00/30.0058 of the Ministry of Education, Youth and Sports of the Czech Republic. The third author (JV) appreciates the support of her department.

\section{REFERENCES}

[1] I. M. Anderson, "Introduction to the variational bicomplex," Contemporary Math., vol. 132, pp. 51-73, 1992.

[2] I. M. Anderson and T. Duchamp, "On the existence of global variational principles," Am. J. Math., vol. 102, pp. 781-867, 1980.

[3] P. Dedecker, "On the generalization of symplectic geometry to multiple integrals in the calculus of variations," in Lecture notes in Math. Berlin: Springer, 1977, vol. 570, pp. 395-456.

[4] P. Garcia, "The Poincaré-Cartan invariant in the calculus of variations," Symposia Mathematica, vol. 14, pp. 219-246, 1974.

[5] H. Goldschmidt and S. Sternberg, "The Hamilton-Cartan formalism in the calculus of variations," Ann. Inst. H. Poincaré, vol. 23, pp. 203-267, 1973.

[6] M. Krbek and J. Musilová, "Representation of the variational sequence by differential forms," Acta Appl. Math., vol. 88, pp. 177-199, 2005.

[7] D. Krupka, "Some geometric aspects of variational problems in fibred manifolds," Folia Fac. Sci. Nat. Univ. Purk. Brunensis, Physica, vol. 14, p. 65 pp., 1973.

[8] D. Krupka, "A geometric theory of ordinary first order variational problems in fibered manifolds, I. Critical sections, II. Invariance," J. Math. Anal. Appl., vol. 49, pp. 180-206, 469-476, 1975.

[9] D. Krupka, "Variational sequences on finite order jet spaces," in World Scientific, Singapore, 1990, pp. 236-254.

[10] D. Krupka, O. Krupková, G. Prince, and W. Sarlet, "Contact symmetries of the helmholtz form," Differential Geometry and its Applications, vol. 25, pp. 518-542, 2007.

[11] D. Krupka and D. Saunders, Eds., Handbook of Global Analysis. Elsevier, 2008.

[12] D. Krupka, D. Saunders, and O. Krupková, "The Cartan form and its generalisations in the calculus of variations," Int. J. Geom. Met. Mod. Phys, vol. 7, pp. 631-654, 2010.

[13] D. Krupka and J. Šeďenková, "Variational sequences and lepage forms," in Diff. Geom. Appl., J. Bureš, O. Kowalski, and D. Krupka, Eds. Prague: Charles University, 2004, pp. 617-627.

[14] P. Olver, Applications of Lie Groups to Differential Equations. New York: Springer, 2000.

[15] A. Trautman, "Invariance of lagrangian systems," in General Relativity; Papers in Honour of J.L. Synge. Oxford: Clarendon Press, 1972, pp. 85-99.

[16] J. Volná and Z. Urban, "The interior Euler-Lagrange operator in field theory," Math. Slovaca, to appear. 
Authors' addresses

\section{Demeter Krupka}

Lepage Research Institute, 78342 Slatinice, Czech Republic

and

School of Mathematics, Beijing Institute of Technology, 5 South Zhongguancun Street, Haidian Zone, Beijing 100081, China

and

Department of Mathematics, University of Ostrava, 30. dubna 22, 70103 Ostrava, Czech Republic E-mail address: demeter.krupka@ lepageri.eu

\section{Zbyněk Urban}

Lepage Research Institute, 78342 Slatinice, Czech Republic

and

Department of Mathematics and Physics, University of Pardubice, Studentska 95, 53210 Pardubice, Czech Republic

E-mail address: zbynek.urban@lepageri.eu

\section{Jana Volná}

Department of Mathematics, Tomas Bata University in Zlin, Nad Stranemi 4511, 76005 Zlin, Czech Republic

E-mail address: volna@fai.utb.cz 